\title{
The effects of gas clouds on converted-wave imaging: A case study from Valhall
}

\author{
Hengchang Dai ${ }^{1}$, Xiang-Yang $\mathrm{Li}^{1}$, and Michael C. Mueller ${ }^{2}$ \\ ${ }^{I}$ Edinburgh Anisotropy Project, British Geological Survey, West Mains Road, Edinburgh, EH9 3LA, UK \\ ${ }^{2}$ BP Amoco Plc, P.O. Box 3092, Houston, Texas 77253-3092 USA
}

\begin{abstract}
Summary
We investigate the effects of gas clouds on P-S converted waves (C-waves) and evaluate practical schemes which compensate for these effects to improve C-wave imaging using the 2D4C dataset from Valhall. The Valhall C-wave data suffer from severe diodic effects (variations with the source-receiver direction) due to gas clouds. These include diodic illumination effect, diodic velocity $\left(V_{c}\right)$ effect, and diodic velocity ratio $\left(\gamma_{\text {eff }}\right)$ effect. Careful processing is required to compensate for these effects. Firstly, the dataset is separated into positive (+) and negative (-) offset data volumes to perform separate velocity analyses. Secondly, since the P-wave velocity is not reliable due to the gas clouds, a data-driven approach, based on optimizing the focus of the $\mathrm{C}$-wave imaging, is required to quantify $\gamma_{\text {eff. }}$ This approach works well in areas free of the diodic illumination effect, and leads to the identification of not only a vertically varying but also a laterally varying $\gamma_{\text {eff }}$ between 2.2 and 2.8 . A simple partition of the dataset is adopted to account for the vertical and lateral variation of $\gamma_{\text {eff }}$ before a full prestack depth migration, and yields an improved C-wave image at Valhall.
\end{abstract}

\section{Introduction}

The Valhall reservoir cannot be well imaged by conventional P-wave techniques due to the effect of a gas cloud (Thomsen et al. 1997). Although P-S converted-waves (C-waves) have been successfully used to image beneath gas clouds in Valhall as well as in other areas (Berg, 1994; Granli et al, 1999), C-waves may still suffer from undesirable effects due to the gas clouds. These side effects, compounded with the asymmetric raypath of the Cwave, will further increase the difficulties and costs in processing. Here, using the Valhall 2D4C dataset as an example, we examine these effects and discuss ways to compensate for these effects during processing.

\section{The effects of gas clouds}

At the edge of a large gas cloud, depending on the source/receiver direction, the (downgoing) P-wave leg of a Cwave arrival may or may not go through the gas cloud (Figure 1). On one hand, if the P-wave leg is going through a gas cloud (-offset, Figure 1), the resultant $\mathrm{C}$-wave will be very weak if not absent; on the other hand, if the $\mathrm{P}$-wave leg is not going through the gas cloud, the resultant $\mathrm{C}$-wave can be very strong. We refer to this as the diodic illumination effect as shown in Granli et al (1999). Figure 2 shows an example of this effect from Valhall. The event (white arrow) in positive offset has strong energy, but it fades in negative offset. If the gas cloud is small and represents a mild velocity variation, the target will still be illuminated from shooting in both positive and negative offsets, but it will result in different stacking velocities $(\mathrm{Vc})$ with source/receiver direction, and this is referred to as the diodic $V_{c}$, as shown in Thomsen (1999). During processing of the Valhall dataset, we also find that $\gamma_{\text {eff }}$ is laterally changing (due to inhomogeneity) and diodic. (More details are discussed in the section on focusing analysis). As a result, it is difficult to select a correct velocity and $\gamma_{\text {eff }}$ suitable for both positive and negative offsets for subsequent processing.

\section{Data processing}

Offset separation. The dataset is separated into positive (+) and negative (-) offset volumes in order to compensate for diodic effects. Figure 3 shows the $\mathrm{C}$-wave images for positive and negative source/receiver direction. Due to the effect of diodic illumination, the target event (between $5 \mathrm{~s}-6 \mathrm{~s}$ ) is not clear between CCP 1150 and 1230 on the negative offset section and between CCP $950-1100$ on the positive offset section. Although the events between 
CCP 850 - 950 appear in both offsets, their shapes are different. We believe this difference is caused by possible diodic variation in $\gamma_{\text {eff }}$. To account for this, a separate focus analysis is required for the two data volumes.

Focusing analysis: $\gamma_{\text {eff }}$ is an important parameter in $\mathrm{C}$-wave processing. It affects the conversion point position, and hence the $\mathrm{C}$-wave stacked image. The lateral variation of $\gamma_{\text {eff }}$ may cause an event to differ on two separate offset images. In order to examine the effect of $\gamma_{\text {eff }}$ variation, we CCP bin the C-wave dataset allowing different $\gamma_{\text {eff }}$ and process each dataset accordingly (CCP scanning). Figure 4 shows the CCP scanning results for shallow events $(0.5 \mathrm{~s}$ $-2.0 \mathrm{~s})$ for both offsets. If $\gamma_{\text {eff }}$ is either too big or too small, the events on the two images with $+/-$ offsets have a different appearance. With a proper $\gamma_{\text {eff }}$ (e.g. 2.5), the events on the two images are nearly identical and focused.

We also perform focusing analysis at the target zone $(4.5 \mathrm{~s}-6.0 \mathrm{~s})$. We subdivide the selected data into two blocks: block 1 - CCPs 900-1000, and block 2 - CCPs 1000-1100. For block 1, a common $\gamma_{\text {eff }}$ equaling 2.2 can be identified for both the positive and negative offset dataset (bottom left, Figure 5). However, for block 2, it is difficult to select a common $\gamma_{\text {eff. }}$ For positive offset $\gamma_{\text {eff }}=2.5$ is the best pick (middle right), whilst for negative offset, there is no obvious choice of $\gamma_{\text {eff }}$.

To sum up, our analysis shows that the events are better focused on the C-wave image with $\gamma_{\text {eff }}$ between 2.2 and 2.5. The image is smeared with $\gamma$ either too large or too small, and a laterally varying and diodic $\gamma_{\text {eff. }}$ is evident from focusing analysis.

Depth- and offset-dependent CCP binning: Full compensation for gas cloud effects requires prestack depth migration which is expensive and needs a precise macro-velocity model. This approach may not be practical in certain circumstances. A simple alternative is to perform a depth- and offset-dependent CCP binning procedure in order to account for lateral and vertical velocity variation followed by DMO and post-stack migration (Zhu, et al, 1999). Here we subdivide the data into five blocks. Separate depth-dependent CCP binning with three vertical zones is performed for these five blocks for both positive and negative offsets. Different Vc's and $\gamma_{\text {eff }} \mathrm{s}$ are used to process the data. The final image is combined from these blocks and is shown in Figure 6. Apart from the blank zone (near the center of the gas clouds), an improved C-wave image of the target is achieved before pre-stack depth migration, which was not obtained on the P-wave image (Figure 7).

\section{Conclusions}

This work examines the diodic effects of gas clouds on C-waves using the Valhall 3D4C dataset. The Valhall data show strong diodic illumination, diodic $\boldsymbol{V}_{\boldsymbol{c}}$ and diodic $\gamma_{\text {eff }}$ and lateral variation in $\gamma_{\text {eff. }}$ Carefully choosing the correct $\gamma_{\text {eff }}$ can improve the C-wave image. A depth- and offset-dependent CCP binning procedure is proposed to compensate for these diodic effects before pre-stack depth migration, and an improved image of the target is achieved.

\section{Acknowledgments}

We would like to thank Olav Barkved and BP Amoco Norge A/S and the Valhall Partners, Amerada-Hess Norge A/S, Elf Petroleum Norge A.S. and Enterprise Oil Norge Ltd. This work is published with the approval of the Director of the British Geological Survey (NERC), and the sponsors of the Edinburgh Anisotropy Project (EAP): Amerada Hess, BP-Amoco, Chevron, Conoco, Elf, ENI-Agip, ExxonMobil, PGS, Phillips, Saga Petroleum, Schlumberger, Shell, Texaco, TotalFina, and Veritas DGC.

\section{References}

Berg, E., Svenning, B., and Martin, J., 1994, SUMIC - a new strategic tool for exploration and reservoir mapping, Expanded Abstracts, $56^{\text {th }}$ EAGE Meeting, Vienna.

Granli, J., Arntsen, B., Solid, A. and Hilde, E., 1999, Imaging through gas-filled sediments using marine shear-wave data, Geophysics, 64, 668-677.

Thomsen, L., 1999, Converted wave reflection seismology over inhomogeneous, anisotropic media, Geophysics, 64, 678-690

Thomsen, L., Barkved, O., Haggard, B., Kommedal, J., and Rosland, B., 1997. Converted wave imaging of the Valhall Reservoir, EAGE Expanded Abstracts, 59, B048.

Zhu, X., Langhhammer, J., King, D., Madtson, E., Helgesen, H. and Brzostowski, M, 1999, Converted-wave pre-stack depth migration of North Sea salt domes Expanded Abstracts, $69^{\text {th }}$ SEG Annual Meeting, Houston, Texas, 1087-1090. 


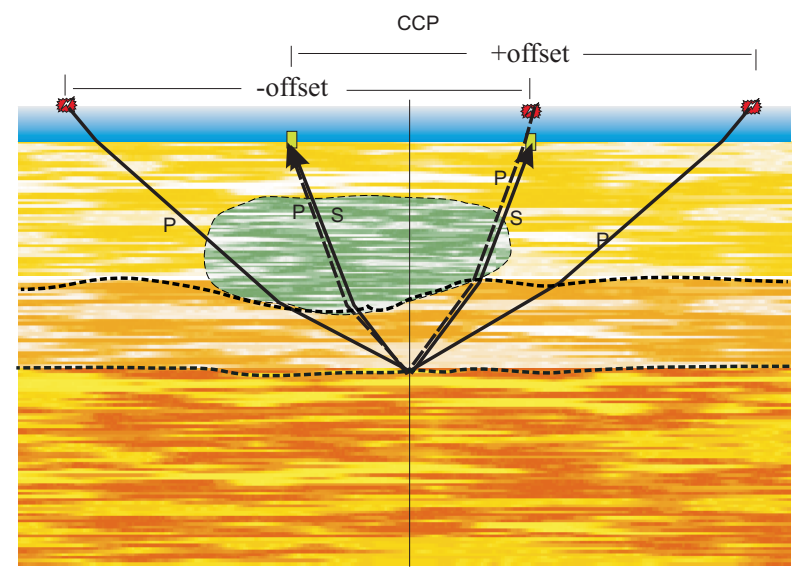

Figure 1. The diodic effects of a hypothetical gas cloud. For Cwaves with -offset, the downgoing $\mathrm{P}$-wave passes through the gas cloud. For $\mathrm{C}$-waves with +offset, the downgoing $\mathrm{P}$-wave does not. The two $\mathrm{C}$-waves have different $\mathrm{V}_{\mathrm{c}}$ and amplitude.

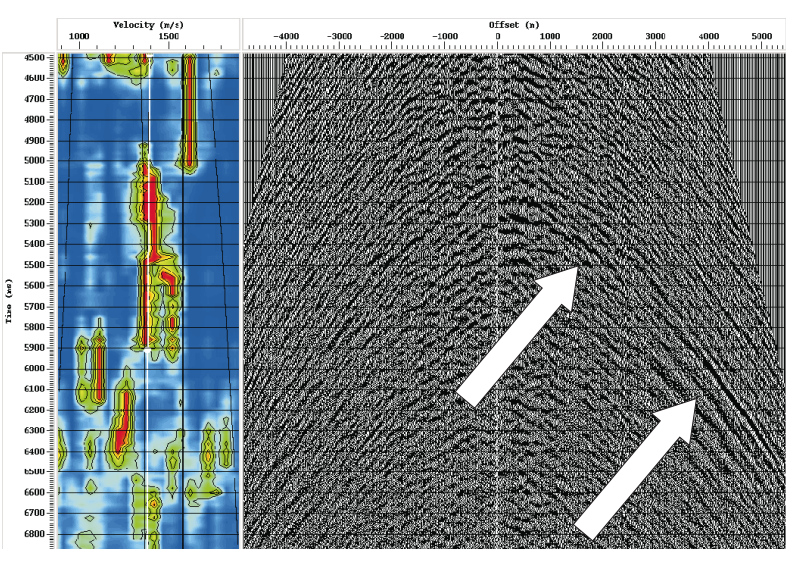

Figure 2. An example showing diodic illumination from the Valhall 2D4C dataset. The right panel is the CCP (1250) gather and the left panel is the velocity spectrum. The event in +offset (right side) is clear, but it fades in -offset (left side).
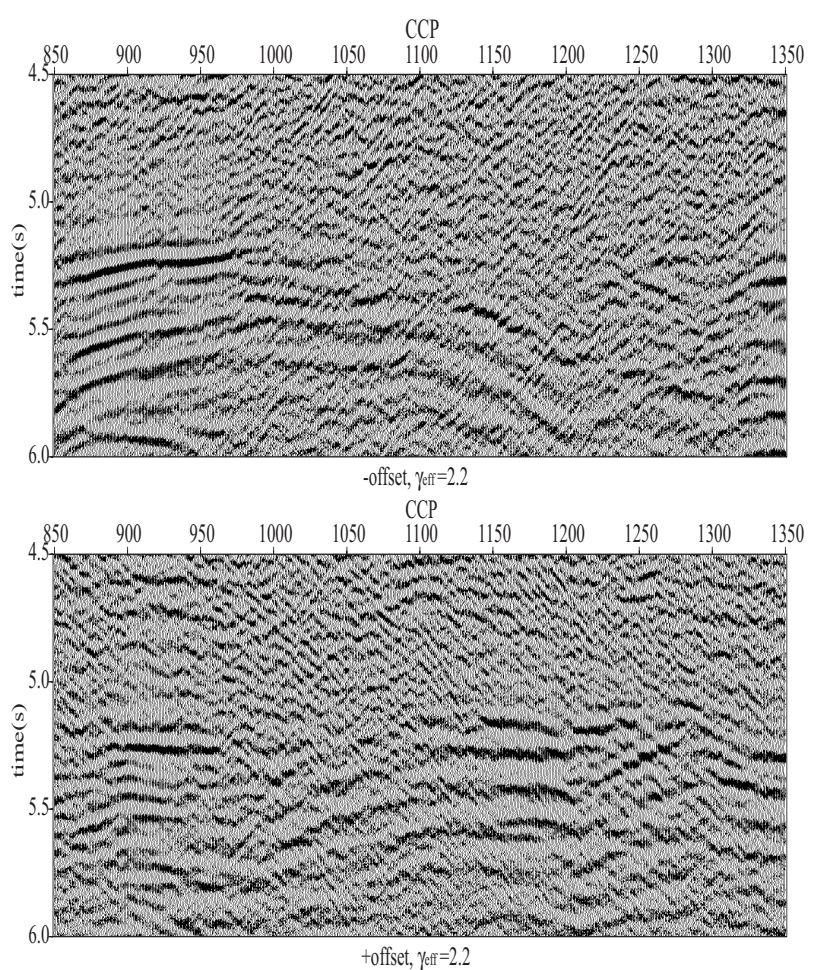

Figure 3. An example of diodic C-wave imaging. The upper panel is obtained from -offset data with $\gamma_{\text {eff }}=2.2$ in which the event on the left is clear. The lower panel is obtained from + offset data with $\gamma_{\text {eff }}=2.2$ in which the event on the right is clear.
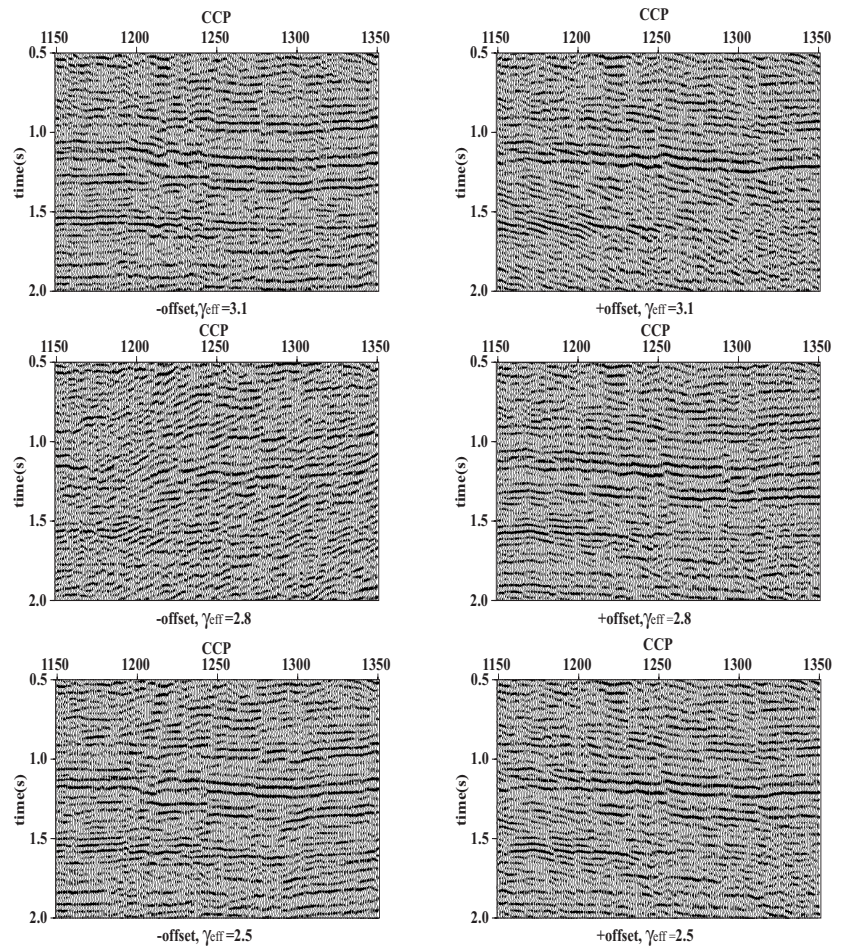

Figure 4. A shallow event obtained from $+/$ - offset with different $\gamma_{\text {eff }}$. The image with $\gamma_{\text {eff }}=2.5$ has the best quality and the events in both offsets are similar and well focused. 

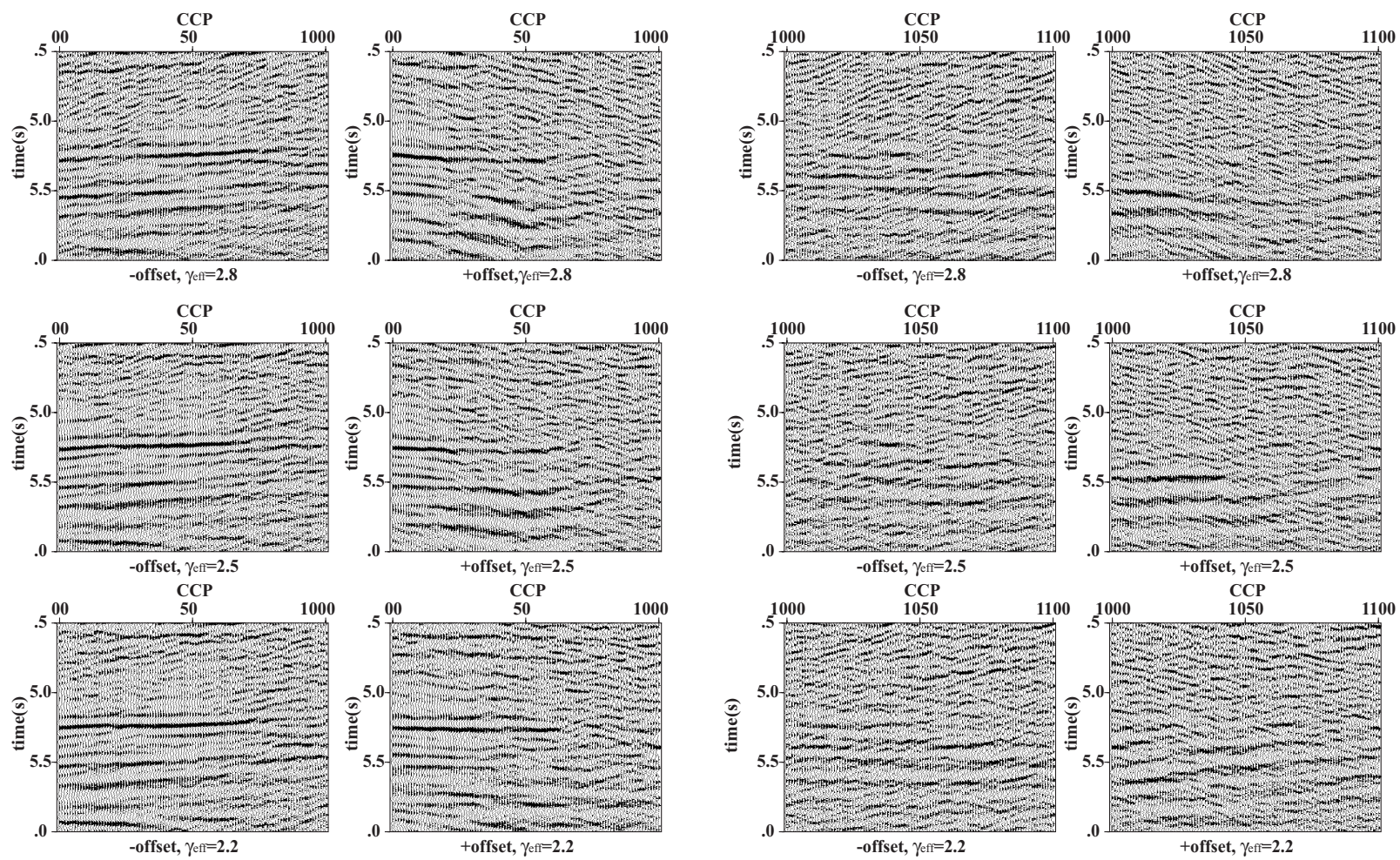

Figure 5. The target (deep) event obtained from (+/-) offset with different $\gamma_{\text {eff: }}$. The events between CCPs $900-1000$ have the better image and is well focused for both offsets with $\gamma_{\text {eff }}=2.2$. owever the events between CCPs $1000-1100$ have a better image and are well focused with $\gamma_{\text {eff }}=2.5$. This shows the lateral changes in $\gamma_{\text {eff. }}$

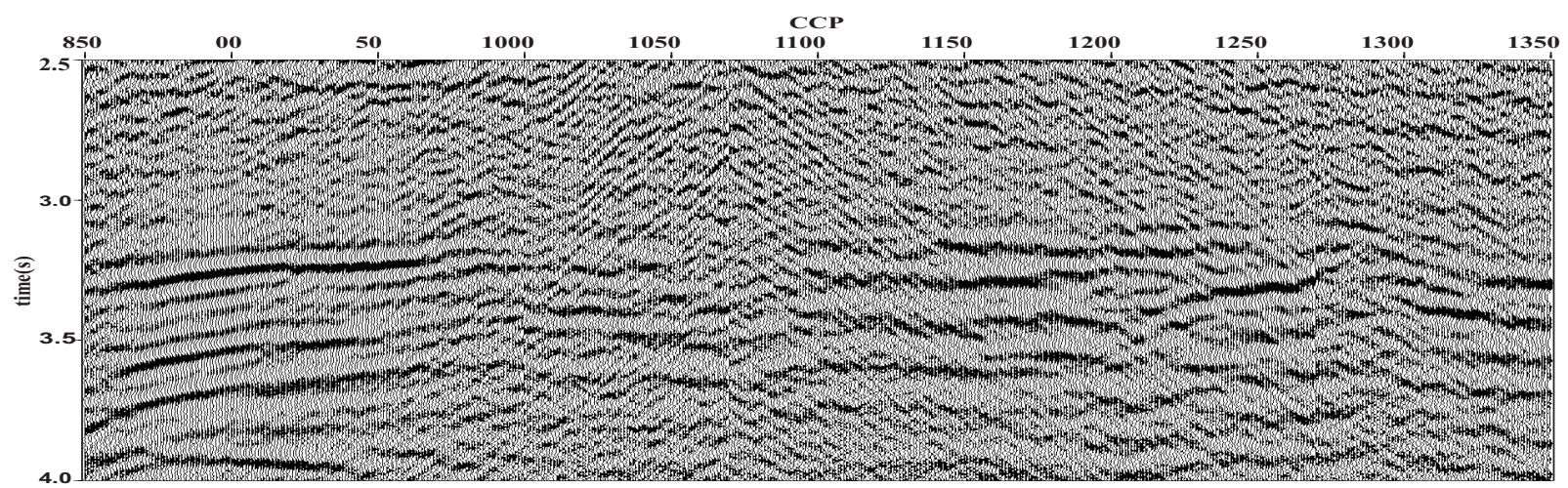

Figure 6. A C-wave image of the target event. The processing considers the effects of diodic Vc and the lateral variation in $\gamma_{\text {eff }}$. The time is converted into P-P time.

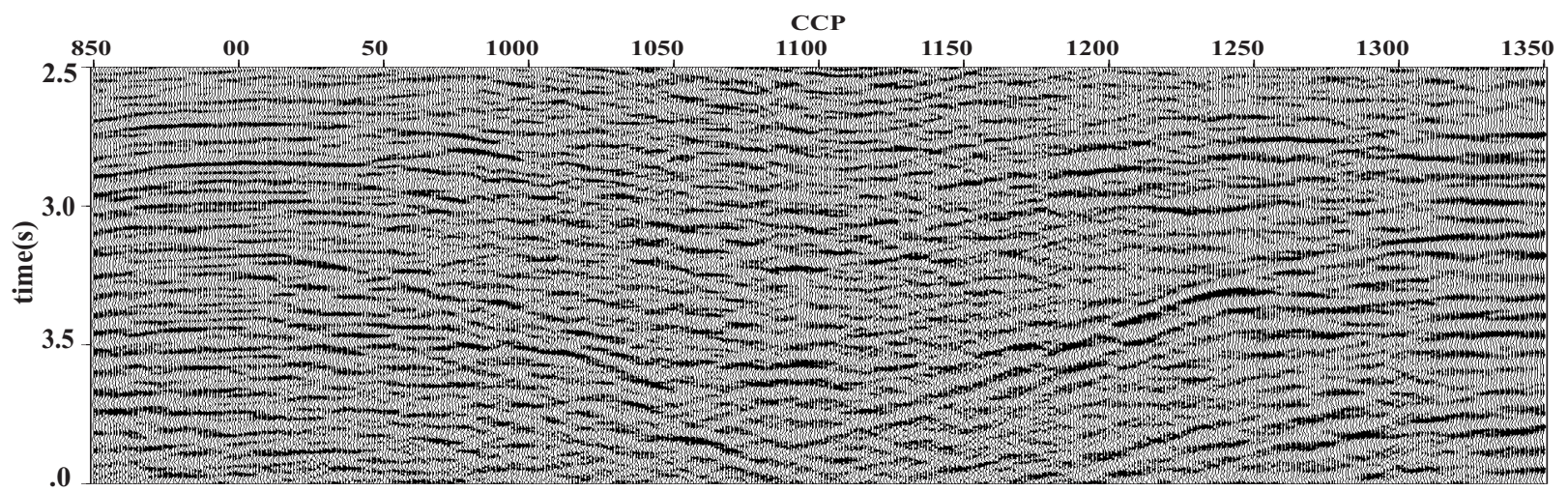

Figure . The P-P image corresponding to the $\mathrm{C}$-wave image of Figure 6 . There is no clear event to indicate the target. 